\title{
Structure and Behavior of AWM and MKW Clusters
}

\author{
Michael Ramuta ${ }^{1}$ \\ Astronomy Department, University of Wisconsin-Madison
}

\begin{abstract}
A grasp of the life-cycles of large-scale structures is critical to understanding the Universe. This can be accomplished through the study of poor clusters-- that is, younger clusters that are likely evolving to another state. The selected clusters are significant in that they are poor but also possess a type-cD galaxy. This brighter central galaxy suggests that these clusters may be dynamically evolved and are potential candidates for fossil groups. In order to more fully understand the structure and behavior of poor galaxy clusters, 13 clusters were selected and analyzed. Using data from the Sloan Digital Sky Survey, we present structural descriptions and mass estimates of these 13 galaxy clusters.
\end{abstract}

\section{Procedure}

The 13 clusters had originally been selected by Morgan, Kayser, \& White (1975) and Albert, White, \& Morgan (1977) in searches for poor clusters with type-cD galaxies. With the SDSS, spectroscopic data was found for 983 galaxies within the 13 clusters, increasing Koranyi \& Geller's count by 572. With right ascension, declination, redshift, and brightness data of galaxies within the clusters, a series of graphs were made to observe substructure. Histograms were made measuring redshift distribution of clusters with fitted Gaussian curves [figure 1]. Clusters were plotted with galaxies colored on a gradient such that the galaxy with the highest velocity was red and the galaxy with the lowest velocity was blue. The large circle represents the half-light radius and the small circle surrounds the type-cD galaxy. To more easily analyze structure, galaxies over select velocities were changed to symbols while the rest remained black dots for contrast [figure 2]. Clusters were then plotted measuring the distance from the type-cD galaxy against the difference from the average velocity of the system [figure 3].

\section{Mass Determination}

Mass Determination: To estimate the mass of the clusters, the half-light radius around the typecD galaxy was used as the effective radius and the velocity dispersion was measured as the standard deviation of the velocities. A mass approximation similar to one used by Heisler \& Tremaine (1985) was used:

$$
M \simeq \frac{5 \cdot \mathrm{R}_{\mathrm{e}} \cdot \sigma_{\mathrm{V}}{ }^{2}}{G}
$$

The results [table 1] should be accurate for the apparently virialized clusters but are less accurate for the clusters not distributed on a guassian.

\footnotetext{
${ }^{1}$ This author would like to acknowledge the support of the National Space Grant College Fellowship Program and the Wisconsin Space Grant Consortium.
} 
MKWJ 2
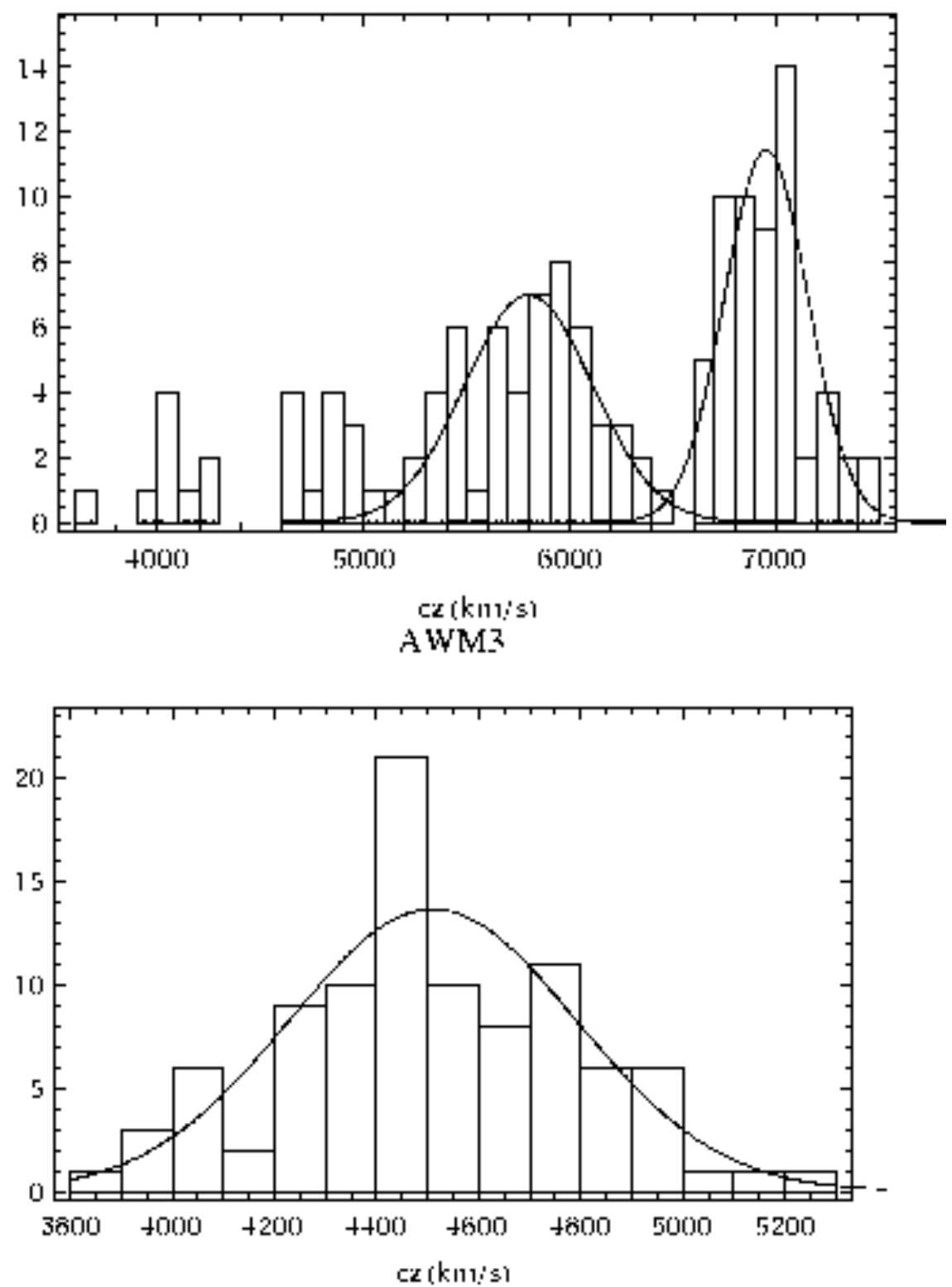

FIGURE 1: Redshift distributions of clusters MKW12 and AWM3 

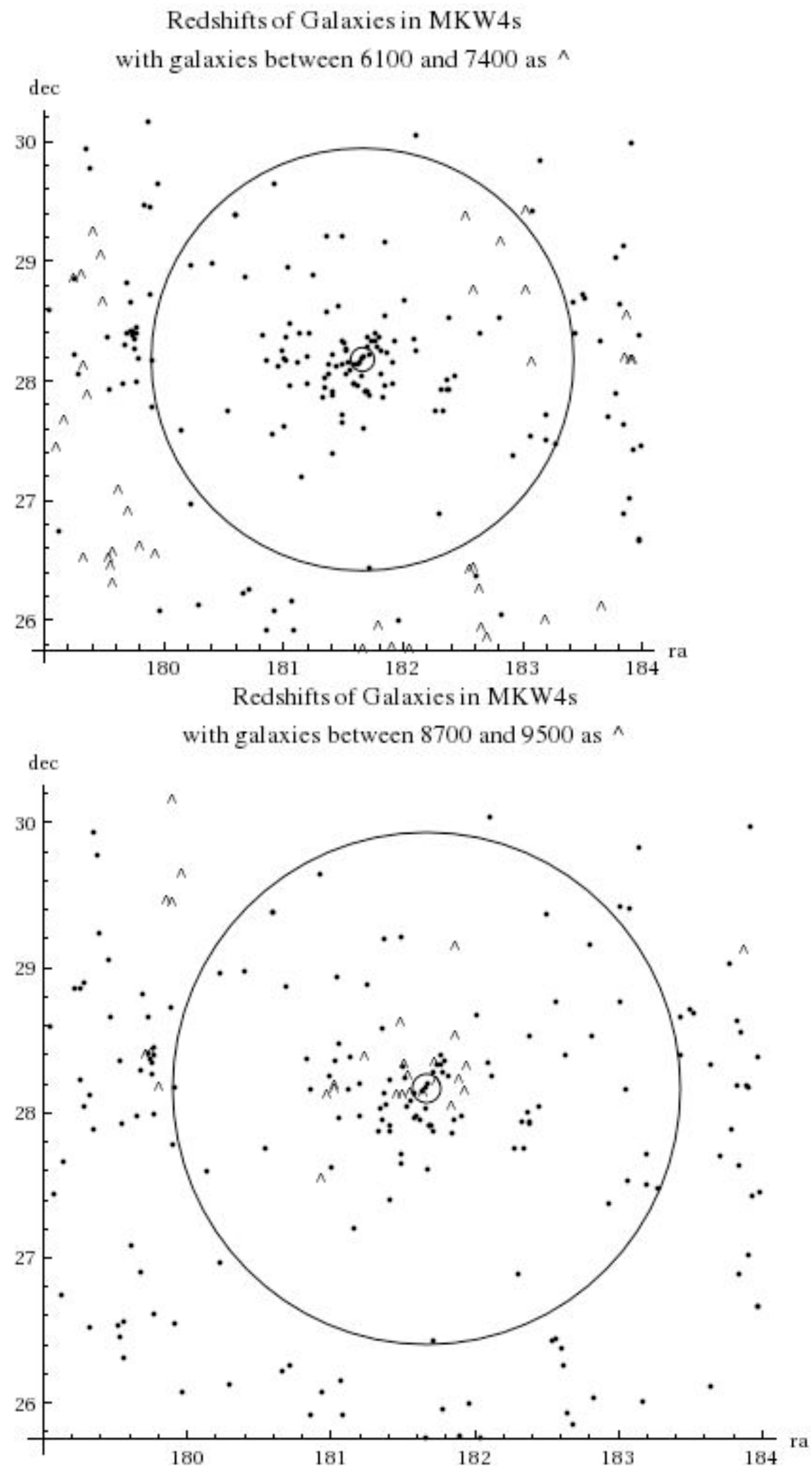

FIGURE 2: Selected Skyplots of MKW4s 
Distance From Center (degrees)

MKW4s

4000

2000

0

$-2000$

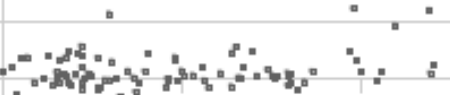

$-4000$
0.5
1.

$\cdot 1,5$

$\therefore$

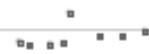

$\therefore$

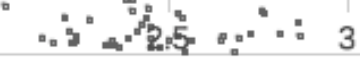

Distance From Center (degrees)

FIGURE 3: Distance from cluster center vs. velocity dispersion of MKW4 and MKW4s

\begin{tabular}{|l|l|l|}
\hline Cluster & $\begin{array}{c}\text { Calculated Mass } \\
\left(\mathbf{1 0}^{\mathbf{1 4}} \text { Solar Masses }\right)\end{array}$ & $\begin{array}{c}\text { Two Estimates by } \\
\text { Jones \& Forman } \\
(\mathbf{1 9 9 9 )} \\
\left(\mathbf{1 0}^{\mathbf{1 4}} \text { Solar Masses }\right)\end{array}$ \\
\hline AWM1 & 3.5 & - \\
\hline AWM2 & .48 & - \\
\hline AWM3 & .69 & - \\
\hline AWM4 & 1.5 & $.23 / .93$ \\
\hline AWM5 & 1.2 & $.78 / 1.14$ \\
\hline MKW1s & .090 & - \\
\hline MKW4 & 1.4 & $.28 / .55$ \\
\hline MKW4s & 14.4 & $.41 / .88$ \\
\hline MKW5 & .31 & - \\
\hline MKW7 & 1.7 & $.48 / .60$ \\
\hline MKW8 & 1.4 & - \\
\hline MKW10 & .091 & - \\
\hline MKW12 & 7.4 & - \\
\hline
\end{tabular}

TABLE 1: Estimated Cluster Masses 


\section{Results}

$A W M 2, A W M 3, M K W 1 s, M K W 4, M K W 5$, and $M K W 10$ all align closely with gaussians and their galaxies are evenly distributed throughout their areas. This implies that these clusters are all in dynamically equilibrium.

$A W M 1$ contains a high velocity tail that implies that it is not yet virialized. This high velocity tail is spread out on the edges of the half-light radius and is likely a sign of ongoing galactic

accretion (Dariush et al., 2007).

$A W M 4$ has a noticeable gap in the center of its redshift distribution as well as a high velocity tail. This high velocity tail all lies centrally in its half-light radius, which indicates continuing accretion.

$A W M 5$ has an unusually uniform distribution of redshift and apparent substructure at the lower right at lower redshifts.

$M K W 4 s$ has a high velocity tail which lies in the center and the upper left which indicates ongoing accretion in these areas. MKW4s also has a low velocity bump which can be seen in a halo which lies right around the half-light radius. This substructure may indicate that MKW4s is a result of two clusters colliding.

$M K W 7$ has both high and low velocity tails that appear to be evenly distributed throughout the cluster.

$M K W 8$ has a high velocity tail which lies in the bottom half of the cluster.

MKW12 fits best with two separate gaussians, meaning MKW12 is probably two colliding clusters. The lower velocity Gaussian is circular shaped in the middle of the plot with a satellite near the top. The higher velocity bump is disk shaped and lies in the upper section of the plot. The galaxies between the gaussians seem to form a bridge between the structures.

\section{Further Research}

Moving forward, the mass estimates must be revised, as there is a systematic error resulting in overestimation. As most of these clusters should be within the search area for the NRAO VLA Sky Survey, the radio data from the survey could help find their radio luminosity functions, yielding a more accurate mass. Further, more analysis of the irregular clusters (AWM5, MKW4s, and MKW12) would provide further information on their structures. 


\section{References}

Albert, C., White, R. \& Morgan, W. 1977, ApJ, 211, 309

Dariush, A., Khosroshahi, H., Ponman, T., et al. 2007, MNRAS, 382, 433

Heisler, J., Tremaine, S., \& Bahcall, J. N. 1985, ApJ, 298,8

Jones, C., \& Forman, W. 1999, ApJ, 511, 65 (JF)

Koranyi, D., \& Geller, M. 2002, AJ, 123, 100

Ledlow, M. J., Loken, C., Burns, J. O., Hill, J.M., \& White, R. A. 1996, AJ, 112,388

Morgan, W.W., Kayser, S. \& White, R.A. 1975, ApJ, 199,545 